\title{
The effect of minocycline on Candida albicans
}

\author{
PAMELA M. WATERWORTH \\ From the Department of Microbiology, University College Hospital, London
}

SYNOPSIS Minocycline, a new tetracycline derivative, was found to inhibit the growth of Candida albicans. Inhibition was much affected by the composition of the medium and was difficult to demonstrate in fluid cultures. Study of the rate of budding in shallow broth cultures in Petri dishes showed that the addition of $20 \mu \mathrm{g} / \mathrm{ml}$ minocycline prolonged the lag phase by three hours. $C$. tropicalis was similarly inhibited and $C$. guilliermondii and $C$. parapsilosis to a lesser degree. Six other tetracyclines were tested and found to inhibit Candida only in very high concentrations.

Minocycline (7-dimethylamino-6-deoxy-6-demethyltetracycline) is the latest semisynthetic to be introduced into Great Britain. It has been found to have equal or slightly greater activity than the earlier tetracyclines against most sensitive species (Martell and Boothe, 1967; Steigbigel, Reed, and Finland, 1968) but is of interest mainly because cross resistance is not complete and many strains of Staphylococcus aureus which are resistant to tetracycline remain sensitive to minocycline.

Minocycline has been available elsewhere for some time and the attention of Dr A. Yeadon of Lederle Laboratories was drawn to the fact that discs containing $30 \mu \mathrm{g}$ minocycline inhibited the growth of Candida albicans. The work reported here was done at his suggestion.

\section{Materials and Methods}

\section{MEDIUM}

Oxoid nutrient broth no. 2 and Oxoid or Lab M nutrient agar (NA) were used for most experiments, but Oxoid blood agar base no. 2 (BAB2) was sometimes included. Carbohydrates were added from a $20 \%$ solution prepared in sterile water.

\section{TEST ORGANISMS}

All the strains of Candida albicans, C. krusei, and C. stellatoides were recently isolated in this department. The strains of $C$. guilliermondii, $C$. tropicalis, Torulopsis glabrata, and C. parapsilosis were kindly supplied by Dr D. W. R. Mackenzie.

\section{ANTIBIOTICS}

Tetracycline, demethylchlortetracycline, chlortetracycline, and minocycline were supplied by Lederle

Received for publication 28 January 1974 .
Laboratories and oxytetracycline, methacycline, and doxycycline were supplied to them by Pfizer Ltd.

MINIMUM INHIBITORY CONCENTRATIONS (MIC) These were determined either by the plate dilution or broth dilution method using double dilutions and a light inoculum.

\section{VIABLE COUNTS}

Viable counts were done by a modified Miles and Misra technique, decimal dilutions being prepared by adding $0.5 \mathrm{ml}$ to $4.5 \mathrm{ml}$ diluent.

\section{RATE OF BUDDING}

Rate of budding was studied using a suspension of C. albicans consisting entirely of single cells (96$98 \%$ ). The organism was grown overnight on a $2 \%$ solution of malt extract solidified with $1 \%$ agar. Growth was harvested, washed twice in sterile water, and re-suspended in sterile water. This suspension was stored at $4^{\circ} \mathrm{C}$ and added to fluid medium with and without minocycline when required. One hundred cells or cell aggregates were then counted at intervals in a haemocytometer chamber.

Incubation in all experiments was at $37^{\circ} \mathrm{C}$ unless otherwise stated.

\section{Effect of Minocycline in Solid Media}

A $30 \mu \mathrm{g}$ disc of minocycline placed on a solid medium always reduced the size of the colonies of $C$. albicans in a surrounding area about $13 \mathrm{~mm}$ in diameter. Inhibition was usually total on both NA, which supported only poor growth of the yeast and NA containing $1 \%$ sucrose, which enhanced growth outside the zone of inhibition considerably. Growth 


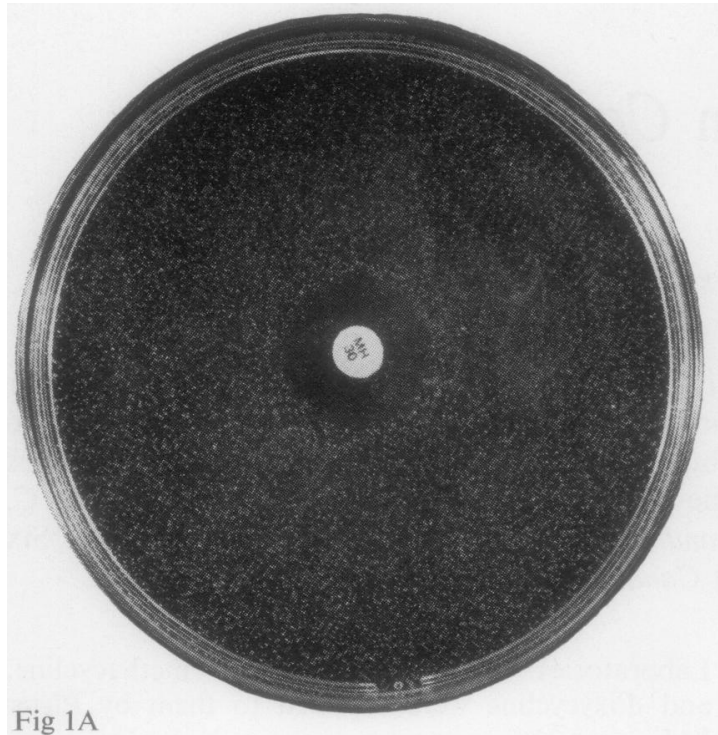

Fig 1 A

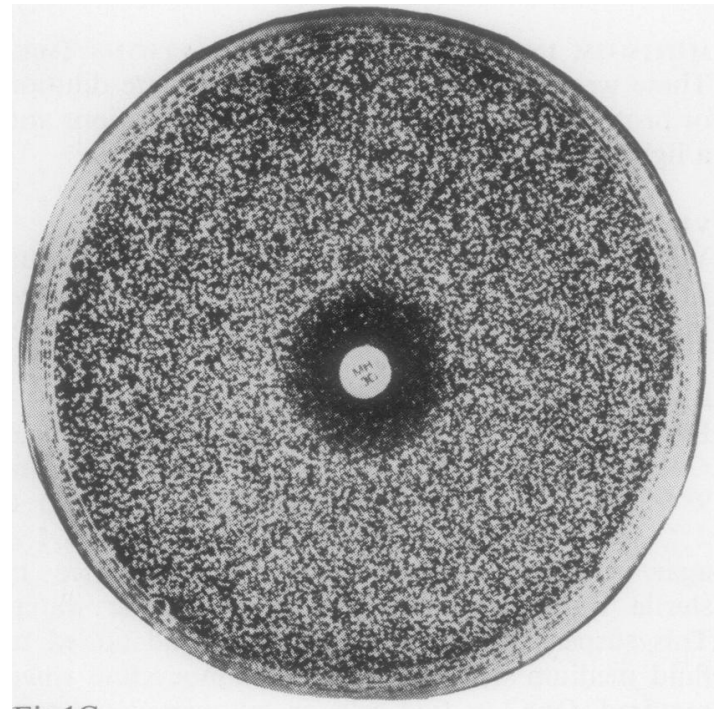

Fig 1C

on BAB2 was better than on NA and in the inhibitory zone minute colonies could be seen only with a lens. The addition of $1 \%$ lactose had no effect but $1 \%$ glucose or maltose greatly enhanced growth and colonies within the inhibition zone were only moderately reduced in size (figure 1 ). If incubation was continued beyond 24 hours colonies appeared in all zones and slowly increased in size, but they seldom reached thesame size as those outside the zone.

As the addition of sucrose improved growth without reducing the inhibitory effect, it was included for most subsequent experiments.

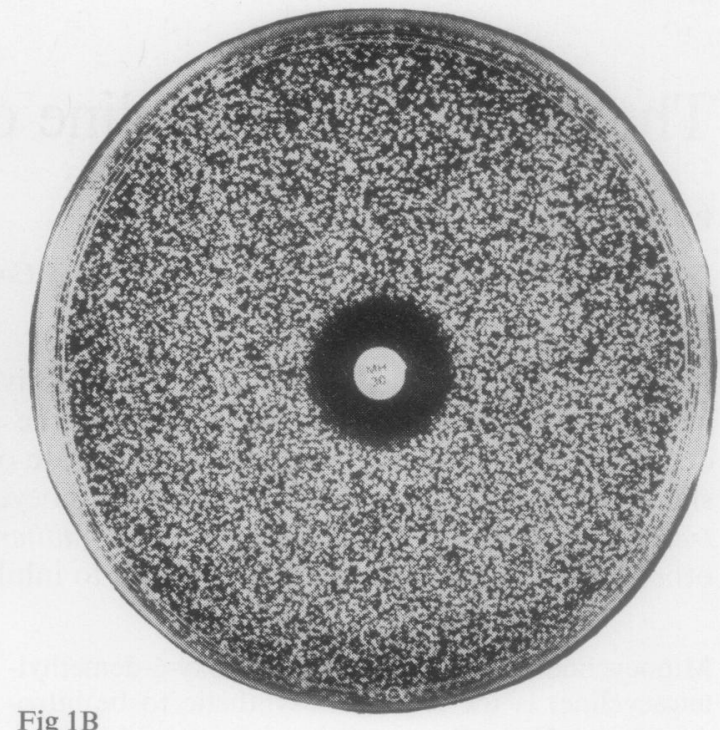

Fig 1B

Fig 1 Effect of minocycline on growth of Candida albicans on (A) nutrient agar (B) $1 \%$ sucrose agar, and (C) $1 \%$ glucose agar. Disc contains $30 \mu \mathrm{g}$.

Whenever there was a growth within the zone of inhibition, this consisted of uniform colonies of diminished size throughout the area and was apparently unaffected by the concentration of the drug. To confirm that this was so, plates of BAB2 with $1 \%$ sucrose and varying amounts of minocycline were inoculated with $C$. albicans to give discrete colonies. After overnight incubation there was some reduction in colony size, on $4 \mu \mathrm{g} / \mathrm{ml}$ and all colonies were much reduced on $8 \mu \mathrm{g} / \mathrm{ml}$, but similar numbers of minute colonies persisted on all concentrations up to and including $256 \mu \mathrm{g} / \mathrm{ml}$. 


\section{Effect of Minocycline on Growth in Fluid Medium}

A number of experiments were done in which broth, sucrose broth, and glucose broth in 3-5 $\mathrm{ml}$ volumes with and without $10 \mu \mathrm{g} / \mathrm{ml} \mathrm{minocycline} \mathrm{were} \mathrm{lightly}$ inoculated with $C$. albicans. The organism grew very poorly in plain broth and moderately and profusely in sucrose and glucose respectively; the addition of minocycline made no obvious difference to growth.

In case this was an effect of lack of oxygen, the experiment was repeated using $7 \mathrm{ml}$ medium in $50 \mathrm{ml}$ flasks in a fast shaking water bath. Profuse and rapid growth appeared in all flasks and viable counts on these and in the previous experiments showed no consistent or significant differences. However, some reduction in the amount of growth resulted from the addition of either 10 or $50 \mu \mathrm{g} / \mathrm{ml}$ minocycline to $10 \mathrm{ml}$ of broth incubated in $9 \mathrm{~cm}$ Petri dishes.

Viable counts on an organism which grows in large clusters are obviously far from satisfactory and Dr D. W. R. Mackenzie suggested that a more reliable indication of the rate of growth might be obtained by observing the rate of budding. Ten $\mathrm{ml}$ volumes of $1 \%$ sucrose broth with and without $20 \mu \mathrm{g} / \mathrm{ml}$ minocycline were lightly inoculated with a single cell suspension of Candida albicans and incubated in Petri dishes. At intervals these were gently agitated and a large loopful was transferred to a counting chamber and the ratio of single and budded cells in the first 100 was noted. The results are given in figure 2 and suggest that the effect of minocycline is simply to prolong the lag phase. However, the similarity in the counts at eight hours should not be taken to indicate similar total growth; from five hours numerous, increasingly large, clusters appeared in the control cultures and growth was obviously much heavier than in the presence of minocycline. Nevertheless growth was never as effectively inhibited in fluid media as it was on solid.

\section{Effect of Minocycline on Other Species of Candida}

Twenty strains of $C$. albicans have been examined and all were found to give a similar zone of inhi-

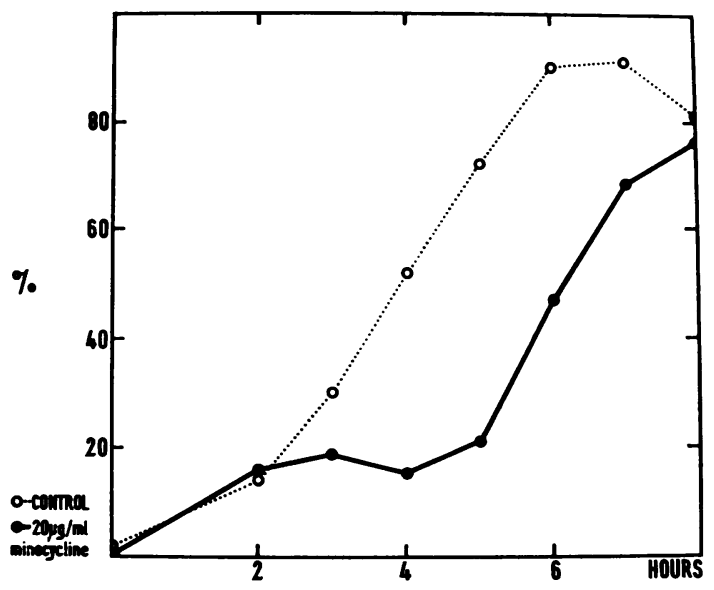

Fig 2 Rate of growth of Candida albicans in $1 \%$ sucrose broth with and without $20 \mu \mathrm{g} / \mathrm{ml}$ of minocycline. Graph shows the percentage of cells with buds.

bition round a cup containing 2 drops of $1000 \mu \mathrm{g} / \mathrm{ml}$ minocycline on either plain or sucrose agar. One strain of $C$. tropicalis was similarly inhibited on NA but colonies of moderate size appeared throughout the zone when sucrose was added. One strain of C. parapsilosis gave a smaller zone which disappeared after 24 hours on the bench and one of C. guilliermondii which grew only moderately well, was only slightly inhibited. One strain each of $T$. glabrata and $C$. stellatoidea and two of $C$. krusei gave barely visible growth and were not inhibited.

The MIC of minocycline for $C$. parapsilosis and C. guilliermondii was determined using $1 \%$ sucrose agar. The results given in table I are surprising; the difference in the size of the zone compared to that of C. albicans would have suggested a greater difference in the MIC of these species.

\section{Effect of Other Tetracyclines}

The MICs of doxycycline, chlortetracycline, demethylchlortetracycline, tetracycline, oxytetracycline, and methacycline for four strains of $C$.

\begin{tabular}{|c|c|c|c|c|c|c|c|}
\hline & Minocycline & Tetracycline & Oxytetracycline & $D M C T$ & Methacycline & Chlortetracycline & Doxycycline \\
\hline $\begin{array}{l}\text { C. albicans (four strains) } \\
\text { C. parapsilosis } \\
\text { C. guilliermondii }\end{array}$ & $\begin{array}{l}8 \\
4 \\
8\end{array}$ & $\begin{array}{r}2048 \\
2048 \\
>2048\end{array}$ & $\begin{array}{l}>2048 \\
>2048 \\
>2048\end{array}$ & $\begin{array}{r}512 \\
512 \\
1024\end{array}$ & $\begin{array}{r}512 \\
512 \\
1024\end{array}$ & $\begin{array}{l}512 \\
512 \\
512\end{array}$ & $\begin{array}{l}256 \\
256 \\
512\end{array}$ \\
\hline
\end{tabular}

Table I Concentrations of various tetracyclines $(\mu \mathrm{g} / \mathrm{ml})$ inhibiting growth on $1 \%$ sucrose agar after 24 hours' incubation $^{1}$

The endpoint was taken as the lowest concentration showing no visible growth to the naked eye. 
albicans and one each of $C$. guilliermondii and $C$. parapsilosis were determined by the plate dilution method using $1 \%$ sucrose agar. The concentrations given in table $I$ are the lowest inhibiting visible growth after 24 hours' incubation, but there was nearly always a marked reduction in colony size at least two dilutions lower and usually some growth one dilution higher after 48 hours' incubation. When this experiment was repeated in broth, the results were complicated by the precipitation of the drugs in the higher concentrations.

\section{Discussion}

Candida spp are generally regarded as highly resistant to all antibacterial antibiotics and there have been many studies suggesting that tetracyclines may even enhance the growth of $C$. albicans (Winner and Hurley, 1964). Minocycline is clearly an exception and relatively quite low concentrations inhibit the growth of this species on solid medium. The significance of this effect is very doubtful as it is much affected by the nature of the available nutrients and degree of aeration. No explanation has been found for the difference between results on solid medium and in broth, or between shaken and stationary aerated fluid cultures. It may be that the degree of aeration is critical, or alternatively, if inhibition is only due to a prolongation of the lag phase, as figure 1 suggests, it may be that the enhanced growth rate in the shaken culture so reduced the lag phase that any prolongation, perhaps also reduced, was missed. It appears that the presence of fermentable carbohydrate interferes with the inhibitory effect of minocycline. None of the species tested were fully inhibited in the presence of glucose, which they all fermented. On the other hand, maximum inhibition of $C$. albicans occurs in the presence of sucrose, which this organism utilizes but does not ferment, and growth of $C$. tropicalis which does ferment sucrose is only partially inhibited. The strains of $C$. parapsilosis and $C$. guilliermondii used fermented sucrose slowly, producing gas only after 48 hours' incu- bation, and inhibition zones with both were less marked than with $C$. albicans.

The sensitivity of $C$. albicans to minocycline may be compared to the peculiar sensitivity of C. tropicalis (also sensitive to minocycline) to polymyxin (Nicholls, 1970). In this case the action of the drug was not significantly affected by the addition of glucose to the medium and the organism was quite rapidly killed.

It seems inconceivable that minocycline could have any effect on established infection with Candida. On the other hand, treatment with tetracyclines is often accompanied by proliferation of Candida spp in the alimentary tract, which is often symptomless, but which may proceed to tissue invasion in the mouth or bowel or even other parts of the body. The mechanism of the effect is uncertain. The suppression of the normal bacterial flora, particularly in the mouth, may simply remove a restraint on the proliferation of small numbers of Candida usually present there. An alternative possibility, referred to above, is that subinhibitory concentrations of tetracyclines may actually stimulate the growth of Candida. Whatever the explanation, it seems reasonable to hope that a tetracycline derivative exerting an inhibitory effect on the growth of C. albicans is less likely to encourage its growth than other derivatives. A clinical trial designed to test the truth of this hypothesis is now in progress.

I would like to thank Dr D. W. R. Mackenzie for his interest and help in this work and in the preparation of the paper.

\footnotetext{
References

Martell, M. J., Jr., and Boothe, J. H. (1967). The 6-deoxytetraclines. VII. Alkylated aminotetracyclines possessing unique antibacterial activity. J. med. Chem., 10, 44-66.

Nicholls, M. W. N. (1970). Polymyxin sensitivity of Candida tropicalis. J. med. Microbiol., 3, 529-538.

Steigbigel, N. H., Reed, C. W., and Finland, M. (1968). Susceptibility of common pathogenic bacteria to seven tetracycline antibiotics in vitro. Amer. J. med. Sci., 255, 179-195.

Winner, H. I., and Hurley, R. (1964). Candida albicans, p. 63. Churchill, London.
} 